\title{
Correction to: Increased average number of medical publications per interviewee from 2009 to 2018: a study of 100 interviewees to an academic gastroenterology fellowship program
}

\author{
Zaid Imam $^{1+}$ and Mitchell S. Cappell ${ }^{2,3^{*+}}$
}

\section{Correction to: BMC Med Educ (2019) 19:402 \\ https://doi.org/10.1186/s12909-019-1841-2}

Following publication of the original article [1], due to miscommunication during proofing, the author notified us the bellow corrections. In this Correction, correct and incorrect versions are shown.

Page 1 Correspondence.

Incorrect: * Correspondence: Zaid.Imam@beaumont.org;

Mitchell.Cappell@beaumont.edu

Correct:"Correspondence:

Mitchell.Cappell@beaumont.edu

Page 2 Methods (paragraph 1).

Incorrect: for endoscopy retrograde.

Correct: for endoscopic retrograde.

Page 3 Statistical analysis (paragraph 1).

Incorrect: years of (instead.

Correct: years (instead.

Incorrect: year) interviews.

Correct: year) of interviewees.

Page 3 Results (Significantly varying parameters with time).

Incorrect: SimplePara>Fig. 1a illustrates.

Correct: Fig. 1a illustrates.

Page 4 Table 1.

Incorrect: Is missing a row in the middle of the table which should be in the table as a second header.

The original article can be found online at https://doi.org/10.1186/s12909019-1841-2

* Correspondence: Mitchell.Cappell@beaumont.edu

${ }^{\dagger}$ Zaid Imam and Mitchell S. Cappell are primary authors.

2Division of Gastroenterology \& Hepatology, William Beaumont Hospital at Royal Oak, MOB \#602, 3535 W. Thirteen Mile Rd., Royal Oak, Ml 48073, USA ${ }^{3}$ Oakland University William Beaumont School of Medicine, Royal Oak, MI 48073, USA

Full list of author information is available at the end of the article
Correct: The corrected Table 1 is presented below.

Page 5 Bottom, right column

Incorrect: 2009 and 2011 vs.2016-2018.

Correct: 2009-2011 vs. 2016-2018.

Page 6 Table 3.

Incorrect: It should have 3 columns and not 1 column. Correct: The corrected Table 3 is presented below

Page 6 Under Table 3 (paragraph 4)

Incorrect: are.

Correct: were.

Incorrect: ten-year-study.

Correct: ten-year-study-period.

Page 7 Author's contributions.

Incorrect: as mentor for Dr. ZI. Both authors read and approved the final manuscript.

Correct: as mentor for Dr. ZI. Both authors are equal and primary authors. Both authors read and approved the final manuscript.

Page 7 Competing interests.

Incorrect: Gastroenterology.

Correct: Gastrointestinal.

\section{Author details}

'Department of Internal Medicine, William Beaumont Hospital, Royal Oak, MI 48073, USA. ²Division of Gastroenterology \& Hepatology, William Beaumont Hospital at Royal Oak, MOB \#602, 3535 W. Thirteen Mile Rd., Royal Oak, Ml 48073, USA. ${ }^{3}$ Oakland University William Beaumont School of Medicine, Royal Oak, MI 48073, USA.

Published online: 27 November 2019

Reference

1. Imam Z, Cappell MS. BMC Med Educ. 2019;19:402. https://doi.org/10.1186/ s12909-019-1841-2. 
Table 1 Comparison of distribution of parameters for interviewees for Gl fellowship: 2009-2011 vs. 2016-2018 using parametric statistical analysis

\begin{tabular}{|c|c|c|c|}
\hline Categorical variables & $\begin{array}{l}\text { Number (\%) with parameter } \\
\text { 2009-2011 (Total } N=48 \text { ) }\end{array}$ & $\begin{array}{l}\text { Number (\%) with parameter } \\
2016-2018 \text { (Total } N=52 \text { ) }\end{array}$ & $P$-value \\
\hline Male (vs. Female Sex) & $34(70.8 \%)$ & $44(84.6 \%)$ & 0.146 \\
\hline Foreign medical graduates (vs. American medical graduates) & $21(43.8 \%)$ & $26(50 \%)$ & 0.554 \\
\hline MD graduates (vs. DO graduates) & $48(100 \%)$ & $46(88.5 \%)$ & 0.027 \\
\hline Applied as chief resident (vs. no chief residency) & $2(4.2 \%)$ & $7(13.5 \%)$ & 0.163 \\
\hline $\begin{array}{l}\text { Medical school affiliated residency program (vs. non-affiliated residency } \\
\text { program) }\end{array}$ & $45(93.8 \%)$ & $45(86.5 \%)$ & 0.322 \\
\hline Residency program in Midwest (vs. elsewhere in United States) & $31(64.6 \%)$ & $27(51.9 \%)$ & 0.228 \\
\hline U.S. Citizen or legal permanent resident (vs. foreign citizenship) & $29(60.4 \%)$ & $35(67.3 \%)$ & 0.535 \\
\hline Numerical variables & $\begin{array}{l}\text { Parameter Mean } \pm \text { S.D. } \\
\text { 2009-2011 (Total } N=48 \text { ) }\end{array}$ & $\begin{array}{l}\text { Parameter Mean } \pm \text { S.D. } \\
\text { 2016-2018 (Total } N=52)\end{array}$ & $P$-value \\
\hline Mean recommendation score (evaluated semi-quantitatively, see Methods) ${ }^{a}$ & $86.96 \pm 1.461$ & $85.1 \pm 0.64$ & $<.0001^{*}$ \\
\hline Mean USMLE Step 1 score & $235.4 \pm 14.1$ & $244.9 \pm 13.5$ & $0.001^{*}$ \\
\hline Mean USMLE Step 2 CK score & $244.9 \pm 16.7$ & $250.8 \pm 15.2$ & $0.069^{*}$ \\
\hline Mean of averaged combined USMLE Step $1 \& 2$ scores $^{b}$ & $240.1 \pm 14.3$ & $246.9 \pm 13.7$ & $0.036^{*}$ \\
\hline Mean number of abstracts (at national meetings) & $1.69 \pm 0.37$ & $7.54 \pm 1.16$ & $<0.0001^{*}$ \\
\hline Mean number of articles (listed in PubMed) & $1.48 \pm 0.30$ & $6.13 \pm 1.29$ & $0.001^{*}$ \\
\hline Mean number of total publications & $3.17+0.48$ & $12.76+1.99$ & $<0.0001^{*}$ \\
\hline
\end{tabular}

N Number, MD Medical doctor, DO Doctor of osteopathy, S.D. Standard deviation, USMLE United States Medical Licensing Examination, CK Clinical Knowledge ${ }^{a}$ Three individuals had missing recommendation scores (see Methods)

${ }^{\mathrm{b}}$ Mean of (step-1 score + step-2 score)/2

*Utilizing independent $\mathrm{t}$-tests assuming unequal variances

Table 3 Correlation coefficient (and $p$-value) between various interviewee parameters and total number of publications for $\mathrm{Gl}$ fellowship per interviewee for 2009-2011 \& 2016-2018

\begin{tabular}{lll}
\hline Parameter & Correlation Coefficient of Parameter with Number of Publications & $p$-value \\
\hline USMLE Step 1 Score & .19 & .06 \\
USMLE Step 2 CK Score & .082 & .42 \\
Average of USMLE & .048 & .64 \\
Steps 1 \& 2 Scores & -.257 & .01 \\
Mean recommendation & & \\
score (see Methods) &
\end{tabular}

